\title{
Gastromalacia (Postmortem Gastric Perforation): Two Cases Reports
}

Dong Ja Kim, SangHan Lee

Department of Forensic Medicine, School of Medicine, Kyungpook

National University, Daegu, Korea

Received: January 21, 2020

Revised: Feburary 10, 2020

Accepted: Feburary 26, 2020

\section{Correspondence to}

SangHan Lee

Department of Forensic Medicine, School of Medicine, Kyungpook

National University, 680

Gukchaebosang-ro, Jung-gu, Daegu

41944, Korea

Tel: +82-53-420-4887

Fax: +82-53-422-4712

E-mail: sanghan1@knu.ac.kr
Gastromalacia refers to the postmortem acute autolytic erosion of the gastric wall. It should be distinguished from gastric perforations caused by antemortem injuries or other pathologic processes, such as ulcer perforations. It appears as a slimy brownish-black perforation of the gastric wall. The diagnosis is based on the presence of a typical macroscopic perforation in the fundic portion of the stomach, lack of vital reactions in the adjacent organs, and lack of clinical or autopsy evidence of trauma. Here, we report two cases of gastromalacia: one in a 53-year-old woman who died from severe head injury and another in a 2-year-old child who died from neglect by his father. Gastromalacia is a welldefined phenomenon, but due to its rarity in the practical work, it could be misdiagnosed as other causes of gastric perforation.

Key Words: Stomach; Autolysis; Artifacts; Postmortem changes
Gastric perforation is uncommonly found during autopsy. This could be caused by perforated gastric ulcers, blunt abdominal traumas, tumors, cardiopulmonary resuscitation, or gastromalacia. Gastromalacia, originally described in the 18th century by John Hunter, is a postmortem artifact caused by the autolysis of gastric tissue due to the release of endogenous enzymes [1]. It is frequently encountered in the fundic portion of the stomach near the gastroesophageal junction. Occasionally, the left leaf of the diaphragm is also perforated by a ragged fenestration, leading to the escape of gastric contents into the pleural cavity. In addition, gastromalacia may also involve the distal esophagus, leading to perforation into the left pleural cavity [2]. These may lead to radiographic findings of pneumoperitoneum or pneumothorax. Here, we present two cases of gastromalacia with a literature review.

A 53-year-old woman was discovered dead in her bedroom by her husband. At the midnight before her death, she had fallen down the basement staircase of a Karaoke room in a drunken state. Subsequently, she had returned to her house and fallen sleep. Her husband stated that she was sleeping when he left for his office at 07:40 but found her dead at 20:40 when he returned home. An autopsy performed the next day revealed a right temporo-parietal subgaleal hemorrhage with 
linear skull fractures, right hemisphere epidural hematoma $(100 \mathrm{~mL})$, basilar skull fractures in the right middle cranial fossa, left temporal lobe contusion, and bluish bruising on the right thigh and buttock (Fig. 1A). In the abdominal cavity, the fundic portion of the stomach and a portion on the left side of the diaphragm showed perforations caused by autolysis (Fig. 1B). The cause of death was traumatic brain damage due to a fall to the right.

A 2-year-old boy died from neglect by his father, who was 22-years-old, divorced, and addicted to playing computer games. He had left his son alone at home for 15 days without care. The whole body of the child had undergone putrefaction but with no evidence of external trauma. On autopsy, no hemorrhages or fractures were observed in the hyoid bone or thyroid cartilage. No brain hemorrhages were observed either. Autopsy revealed a rupture of the gastric fundus containing $50 \mathrm{~mL}$ of undigested food material (Fig. 2). There was no leakage of gastric contents into the abdominal cavity or evidence of peritonitis. The gross appearance was typical of gastromalacia. Histological examination was not performed.

In these reported cases, the diagnosis was based on characteristic autopsy findings. Typical findings were the fundic location, slimy gelatinous thinning of the gastric wall, the lack of vital reactions in the surrounding tissues and organs, and no evidence of trauma.

Gastromalacia should be considered in the differential diagnosis of pneumoperitoneum or pneumothorax on postmortem radiography [3]. In addition, forensic pathologists should exercise caution in the differential diagnosis of gastric ruptures followed by stress-induced or chronic peptic ulcers and other traumatic ruptures [4]. Characteristic features of gastromalacia are unusual macroscopic findings of gastric perforation and the site of rupture because most peptic ulcer perforations involve the lesser curvature of the antrum, close to the incisura angularis. Pathologic findings of peptic ulcer are necrosis, inflammatory reaction, granulation tissue, and fibrosis. Stress-induced ulcer perforation usually causes gastrointestinal bleeding. Findings of peritoneal

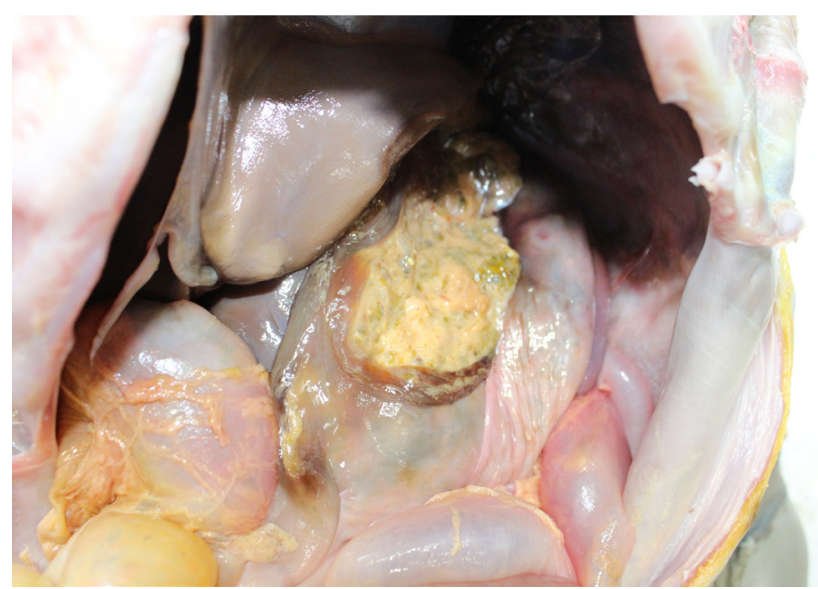

Fig. 2. A photography of the upper abdominal content showed a large defect in the gastric fundus without evidence of surrounding peritonitis. Undigested gastric contents were present.

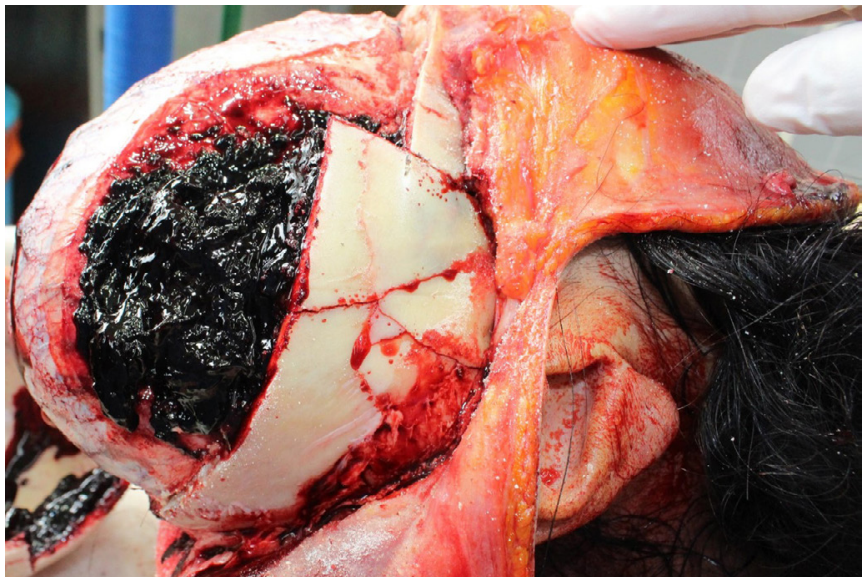

A

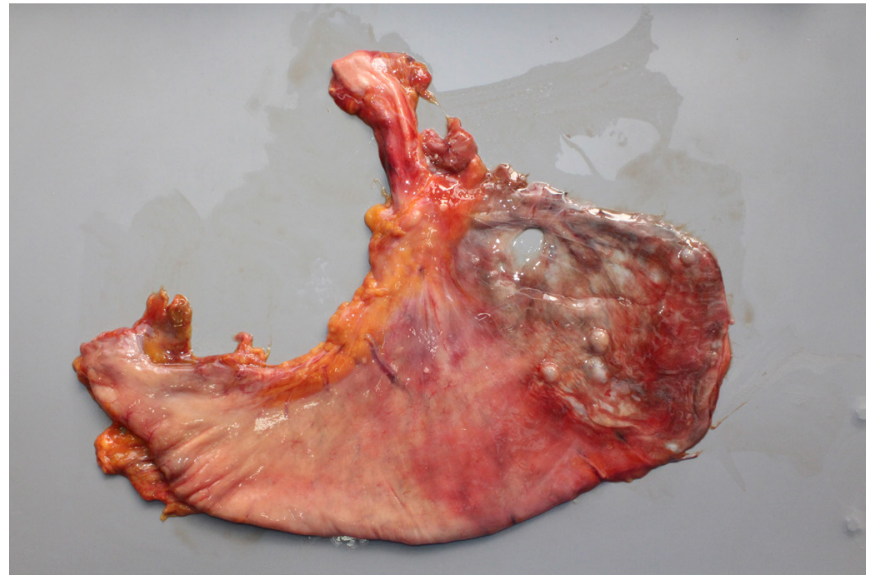

B

Fig. 1. (A) Large amount of epidural hematoma $(100 \mathrm{~mL})$ and linear skull fractures were seen in right brain hemisphere. (B) The gastric wall showed perforation by autolysis in fundic portion. 
inflammatory reactions, such as fibrin formation, abscess formation, or adhesion, were not present in our cases. Gastromalacia is potentially facilitated by bacteria but is not associated with an inflammatory response of the affected gastric wall or adjacent peritonitis [5]. In cases of traumatic rupture, an isolated gastric perforation is unusual, and gastric perforation is strongly associated with injuries of the adjacent organs, including rib fractures and splenic or renal injuries [6,7]. In cases of infant or child death, gastromalacia should be distinguished from abusive injuries.

Gastromalacia is observed more often in cases of a closed head injury and is related to stimulation of the heat regulatory center in the brain and a terminal surge in body temperature, promoting autolysis [2]. Severe head injuries appear to increase particularly the pancreatic polypeptide release by influencing the autonomic centers in the midbrain, which may be related to postmortem gastromalacia [8]. The timing of gastric perforation due to gastromalacia has not been clearly defined, but Laczniak et al. [6] reported that perforation could occur as early as 20 hours after death. In case 1 , autopsy was performed approximately 26 hours after the estimated time of death, so gastromalacia likely occurred within a short time period after death. In case 2 , the timing of gastric perforation was impossible to estimate because of severe putrefaction.

In conclusion, gastromalacia is a well-recognized artifact in autopsy, and doctors of forensic medicine must be aware of this postmortem finding in order to avoid misdiagnosis. In particular, pneumoperitoneum or pneumothorax on postmortem radiography must be interpreted with caution.

ORCID: Dong Ja Kim: https://orcid.org/0000-00018462-3173; SangHan Lee: https://orcid.org/0000-00030390-3494

\section{Conflicts of Interest}

No potential conflict of interest relevant to this article was reported.

\section{References}

1. Hunter J. On the digestion of the stomach after death. Philos Trans R Soc Lond 1772;62:447-52.

2. Perper JA. Time of death and changes after death. In: Spitz WU, ed. Medico-legal investigation of death: guidelines for the application of pathology to crime investigation. 4th ed. Springfield, IL: Charles C Thomas; 2006. p. 14-49.

3. Usui A, Kawasumi Y, Hosokai Y, et al. Postmortem radiography of gastromalacia: case reports. Jpn J Radiol 2013;31:637-41.

4. De-Giorgio F, Lodise M, Pascali VL, et al. An unusual case showing fatal rupture of a gastric ulcer or gastromalacia? The importance/role of histology for differential diagnosis.J Forensic Sci 2015;60:240-2.

5. Bell LG, Thomas EE Jr, Skillicorn SA. Gastromalacia; a review and report of one case with recovery. Ann Surg 1956;143:106-11.

6. Laczniak AN, Sato Y, Nashelsky M. Postmortem gastric perforation (gastromalacia) mimicking abusive injury in sudden unexplained infant death. Pediatr Radiol 2011;41:1595-7.

7. Tejerina Alvarez EE, Holanda MS, Lopez-Espadas F, et al. Gastric rupture from blunt abdominal trauma. Injury 2004;35:228-31.

8. Larson GM, Koch S, O'Dorisio TM, et al. Gastric response to severe head injury. Am J Surg 1984;147:97-105. 